\title{
Microreview
}

\section{The metabolic control of schistosome egg production}

\author{
Edward J. Pearce* and Stanley Ching-Cheng Huang \\ Department of Pathology and Immunology, Washington \\ University School of Medicine, St. Louis, MO \\ 63110-1093, USA
}

\section{Summary}

Schistosomiasis is a neglected tropical disease caused by infection with trematode parasites of the genus Schistosoma. Despite ongoing treatment programmes, the prevalence of schistosomiasis has failed to decline and the disease remains a cause of severe morbidity in millions of people. Understanding the biology of egg production by schistosomes is critical since eggs allow transmission of the infection, and when trapped in host tissues induce the immune responses that are responsible for the pathologic changes that underlie disease development. Unusually among trematodes, adult schistosomes exhibit sexual dimorphism and display a fascinating codependency in that the female is dependent on the male to grow and sexually mature. Thus, virgin females are developmentally stunted compared with females from mixed-sex infections and are unable to lay eggs. Moreover, fecund female schistosomes rapidly lose the ability to produce eggs when placed in tissue culture. Here we discuss the metabolic regulation of egg production in schistosomes, and in particular the critical role played by fatty acid oxidation in this process.

\section{Introduction}

Infection with trematode flatworms of the genus Schistosoma causes chronic and debilitating disease in over 200 million people worldwide (Chitsulo et al., 2004; King and Dangerfield-Cha, 2008). Adult S. mansoni worms live within the mesenteric veins producing eggs that are intended to pass into the intestinal lumen for release into the environment to continue the life cycle

Received 6 February, 2015; revised 27 March, 2015; accepted 2 April, 2015. *For correspondence. E-mail edwardpearce@ @ath.wustl.edu; Tel. (314) 286 2518; Fax (314) 3629108. and allow transmission of the infection (Pearce and MacDonald, 2002). However, because blood within the portal vasculature flows away from the intestine, many eggs are carried to the liver, where they become trapped in sinusoids, and elicit strong Th2 cell-mediated immunopathology which is the cause of disease manifestations (Pearce and MacDonald, 2002). Since egg production is key for both transmission and pathogenesis, studying the mechanisms involved in schistosome reproductive development could lead to new methods of preventing or treating disease (LoVerde, 2002). Reproduction is a bioenergetically demanding process for female schistosomes, which each can produce 300 or more eggs per day (depending on species). Here we will focus on what is known about the metabolic regulation of egg production by these parasites.

Unusually among parasitic trematodes, adult schistosomes exhibit sexual dimorphism and display a fascinating codependency: the female resides in a groove (the gynecophoric canal) on the ventral side of the male, and ongoing physical pairing (but not sperm transfer; Basch and Basch, 1984) is necessary for proper sexual development (Armstrong, 1965; Michaels, 1969; Erasmus et al., 1982; Basch and Basch, 1984; Popiel et al., 1984b; Shaw, 1987; Kunz, 2001). Unmated adult female schistosomes, from female-only infections, are developmentally stunted compared with females from mixed-sex infections and are unable to lay eggs (Kunz, 2001; Grevelding, 2004). Furthermore, egg-laying females that are physically separated from their partners and are surgically implanted into a host in the absence of male worms cease egg production and regress reproductively to an immature state. Interestingly, this regression is reversible because normal reproductive activity is resumed when separated females are re-paired with males (Erasmus, 1973; Popiel and Basch, 1984a; Kunz, 2001). Much of the change in overall size of a female worm as it sexually matures or regresses is due to changes in the vitellarial tissues. The vitellarium is a proliferative tissue that occupies the posterior two-thirds of the female and produces cells that surround the ovum and provide the precursor proteins for eggshell formation and nutrients for the developing embryo. There is evidence that vitellarial involution in separated female parasites, and the failure of the vitellarium to develop in virgin females, is due to a lack of 
immature vitellocyte proliferation under these conditions (Den Hollander and Erasmus, 1984; Knobloch et al., 2002), or to profound increases in mature vitelline cell apoptosis (Galanti et al., 2012), or perhaps to a combination of both. While clearly sufficient to allow and sustain female development in vivo, male parasites are insufficient to prevent vitelline cell apoptosis, vitellarial atrophy or female sexual regression in vitro, suggesting that an additional factor(s) present in the host, but absent in tissue culture, is playing a critical role in female reproductive tract health (Galanti et al., 2012). This is an issue, since the absence of a culture system for schistosomes that fully supports the production of viable eggs by female worms represents a roadblock to the detailed molecular study of these parasites (Kunz, 2001).

\section{Female schistosome maturation and fecundity: a matter of correct nutrition?}

Mating-associated changes in vitelline cell proliferation and apoptosis are intriguing because differing metabolic pathways are argued to preferentially favour cellular proliferation versus cellular longevity in other systems (Pearce, 2010). There have been numerous suggestions that male parasites promote female maturation by 'providing' key nutrients (e.g. Gupta and Basch, 1987). The fact that starvation in planaria (free living flatworms) can lead to reversible reproductive system involution through apoptosis is consistent with the possibility that vitelline cell loss is the end result of nutritional deprivation in female parasites (Hyman, 1951; Pellettieri et al., 2010). This view of vitellarial involution is compatible with the observed effects of tissue culture on paired and unpaired females, since it is conceivable that regardless of the presence of male parasites, culture conditions are failing to provide key nutrients that would normally be available in vivo. This is consistent with the views of Paul Basch, who went to great lengths to create a complex medium that could support the development and maintenance of fecund female schistosomes (Basch, 1981) (success eluded him in this endeavour), that the inability of unpaired worms to produce eggs is a reflection of the fact that they are undernourished (Gupta and Basch, 1987). It seems reasonable that male parasites may serve to provide a signal that allows female parasites to access key nutrients, and that this process is of value only if the nutrients are present in the environment. (We will not specifically address the nature of this male-derived signal in this review.)

Fecund female worms from mixed-sex infections can produce eggs immediately ex vivo, but lose the ability to do so over the course of several days in vitro. We postulate that this reflects the use and final exhaustion of stored metabolic resources. The most likely metabolic stores in schistosomes are glycogen and fat. Glycogen is a source of glucose, but glucose is present in excess in tissue culture, and can be used for survival by worms in this setting (Schiller et al., 1975), and so the depletion of glycogen stores seems unlikely to be a critical factor in vitro (or in vivo). Moreover, $<3 \%$ of the dry weight of female schistosomes is made up of glycogen, but these worms take up three times their dry weight of glucose each day, supporting the view that glucose is used directly and not to establish energy stores in the form of glycogen (Skelly et al., 2014). Rather, lipid stores are most likely to be critical here. Schistosomes possess considerable triacylglycerol stores when recovered from mice (Brouwers et al., 1997), but the function of these stores has remained unclear (Berriman et al., 2009). Available evidence, discussed in detail below, indicates that these triacylglycerol stores are an essential intermediate in the metabolic pathway that supports egg production. Schistosomes are unable to synthesize fatty acids from other substrates (Meyer et al., 1970), but can take up fatty acids from the environment and store them as triacylglycerols (Rumjanek and Simpson, 1980; Young and Podesta, 1982). Female schistosomes ingest large amounts of lipid (50\% of their body mass per day) (Skelly et al., 2014). Fatty acid uptake is believed to occur across the gut surface; saposin homologues have been identified in the gut lumen in schistosomes, supporting this as the major route of uptake (Don et al., 2008; Skelly et al., 2014). Intriguingly, the fecundity of schistosomes is dramatically increased when, experimentally, their hosts are fed with high-fat diets (Neves et al., 2007). Taken together, we believe that these observations support the view that fat is an essential nutrient for egg production by schistosomes.

\section{Oxidative phosphorylation (OXPHOS) is a critical pathway for schistosome egg production, but not for schistosome survival}

Glucose, fatty acids and amino acids are important macronutrients because they are used to fuel the production of ATP. The two major pathways that generate ATP are glycolysis, fuelled by glucose, and OXPHOS, which is coupled to the TCA cycle, which can be fuelled by glucose, fatty acids and glutamine (Fig. 1). ATP production by glycolysis can occur in low or absent oxygen, and under these conditions is referred to as Warburg metabolism (Warburg, 1956). In addition to generating energy, these pathways allow the production of key intermediates for biosynthesis. There is a general view that there is no appreciable lipid or amino acid catabolism in helminth parasites (Barrett, 2009) and glycolysis is considered to be the essential source of ATP in the intramammalian stages of schistosomes (Schiller et al., 1975; Barrett, 


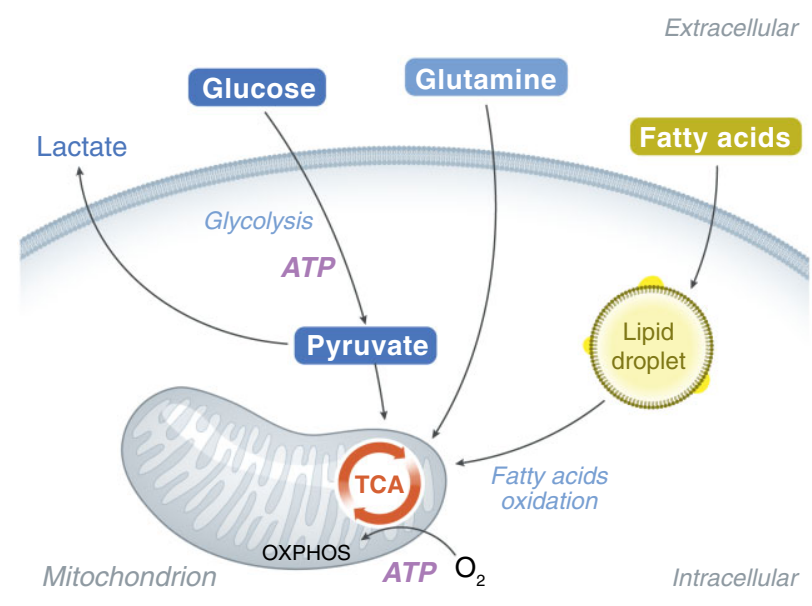

Fig. 1. Glycolysis allows the import of glucose and its conversion into pyruvate in the cytosol. Pyruvate has two possible main fates. The first is conversion into lactate. In this process, $\mathrm{NAD}^{+}$is produced which can be reused for the anaerobic production of ATP by glycolysis (Warburg metabolism). Alternatively, pyruvate enters mitochondria where it is converted into acetyl-CoA which enters the TCA cycle. Fatty acids and glutamine can also be utilized via the TCA cycle as indicated. Fatty acids are used for the synthesis of triacylglycerols, which can be stored in lipid droplets.

Triacylglycerols are broken down by a regulated process of lipolysis to release fatty acids for oxidation. The TCA cycle fuels OXPHOS and the oxygen-dependent production of ATP.

2009) (although cercariae, the free living infectious stage, primarily use OXPHOS; Horemans et al., 1991). Published data supporting the view that adult schistosomes are dependent on glucose are extensive, and include, for example, those in a paper by Schiller et al. (1975) which over 35 years ago reported that schistosomes can survive in vitro in anaerobic conditions as long as glucose is present. Under these conditions, mitochondrial OXPHOS cannot occur and the worms must be using Warburg metabolism to generate energy. Interestingly, Schiller et al. (1975) reported that worms placed under anaerobic conditions in vitro rapidly ceased egg production. This contrasts with aerobic cultures where female schistosomes continue to produce viable eggs for several days ex vivo (Michaels and Prata, 1968; Galanti et al., 2012; Rinaldi et al., 2012). One interpretation of these data is that schistosomes can survive using Warburg metabolism but need to use OXPHOS to produce eggs, a metabolic process that they cannot maintain in tissue culture. There are convincing data to support the view that fecund female schistosomes are using OXPHOS in vivo, and immediately ex vivo (van Oordt et al., 1985; Huang et al., 2012), but lose the ability to do so over time in vitro (Schiller et al., 1975; Huang et al., 2012). We speculate that this reflects the fact that schistosomes can survive by using Warburg metabolism, but are capable of using OXPHOS and that the process of egg production is dependent on this oxygen-dependent form of metabolism.
In other words, there may be tissue-specific metabolic programming in female schistosomes, such that all cells use glucose through glycolysis and OXPHOS to a greater or lesser extent, but that among these cells, only mature vitellocytes have an additional and absolute requirement for OXPHOS to survive.

\section{Fatty acid oxidation is required for egg production}

Despite the ongoing belief that schistosomes cannot utilize fatty acid (or $\beta$-) oxidation to support OXPHOS (Barrett, 1981; Ferreira et al., 2014a,b), the schistosome genome nevertheless encodes the enzymes of the $\beta$-oxidation pathway, through which fatty acids are catabolized into the TCA cycle (Berriman et al., 2009), and mitochondrial oxygen consumption in schistosomes can be inhibited by etomoxir, a drug that blocks the transfer of activated fatty acids into mitochondria for subsequent oxidation (Huang et al., 2012). Moreover, genetic loss of function of Acyl CoA synthase and Acyl CoA dehydrogenase, key enzymes in the $\beta$-oxidation pathway, also results in diminished mitochondrial oxygen consumption. Most importantly from the perspective of the topic under discussion here, all of these interventions, along with pharmacological approaches for blocking OXPHOS, have a marked inhibitory effect on egg production by female parasites recently placed in tissue culture (Huang et al., 2012).

The understanding of how fatty acids are utilized by cells is developing rapidly. Following acquisition from the environment, fatty acids are converted into triacylglycerols and stored in cytoplasmic lipid droplets (LDs), from which they are released in a regulated fashion by lipolysis (Fig. 1) (Guo et al., 2009; Zechner et al., 2012). Fatty acids released in this way are used to fuel $\beta$-oxidation, but also act as endogenous ligands for nuclear receptors that induce the expression of genes encoding the $\beta$-oxidation pathway, and that regulate mitochondrial biogenesis (Palanker et al., 2009; Haemmerle et al., 2011). Thus, there is a link between LD and mitochondrial numbers and activity. Interestingly, greater than $40 \%$ of the lipid in adult schistosomes is in the form of triacylglycerol (Brouwers et al., 1997) and stains for triacylglycerols have revealed that fecund female schistosomes possess a remarkable number of LD within their vitellarial tissues (Huang et al., 2012). There are significantly fewer LDs in virgin females, and in previously fecund females that have ceased to produce eggs as a result of being cultured (Huang et al., 2012). Moreover, mitochondrial oxygen consumption declines greatly as female schistosomes are maintained in tissue culture medium (Huang et al., 2012). Thus, there are dynamic and kinetically coupled changes in LD and mitochondrial oxygen consumption that are related to 
changes in female schistosome fecundity ex vivo. This is intriguing in the context of our understanding of the biology of the insect fat body (Arrese and Soulages, 2010). In insects, the fat body is an organ that partially surrounds the intestine and reproductive organ, and is a major site of triacylglycerol storage in cytoplasmic LD. The fat body plays a critical role in bioenergetically demanding processes such as flight and reproduction and in allowing survival during periods of starvation. While platyhelminths do not have fat body organs, it is interesting to speculate that the LD complex in vitellarial cells serves an analogous function to the fat body at least insofar as reproduction is concerned and that vitelline cell functions require them to utilize fatty acids, via a pathway that includes their storage in LD and presumably release by regulated lipolysis (Zechner et al., 2012).

In mammals, transcriptional activation of genes regulating fatty acid oxidation and controlling mitochondrial biogenesis is mediated to a considerable extent by the PPAR nuclear receptors (Plutzky, 2011). The identity of physiologic ligands for these receptors is intensely debated. However, an exciting recent publication has shown that the lipolysis of LD leads to the production of endogenous fatty acids that are ligands for PPAR $\alpha$ (Haemmerle et al., 2011). Invertebrates lack PPARs, and work in Drosophila and Caenorhabditis elegans indicates that in these organisms, lipid mobilization and $\beta$-oxidation are regulated by a related nuclear receptor, HNF4. For example, in Drosophila, HNF4 null mutants are unable to use their lipid reserves even when starved, and exhibit reduced expression of genes controlling lipid catabolism and $\beta$-oxidation (Palanker et al., 2009). In this feedforward model, HNF4-induced increases in $\beta$-oxidation allow LD lipolysis to occur, and in the absence of HNF4, LD resources cannot be utilized even during starvation. The schistosome genome is recognized to encode at least 21 nuclear receptors including an HNF4 homologue (Wu and Loverde, 2008; Wu and LoVerde, 2011). Intriguingly, HNF4 expression is regulated through the intramammalian life stages, peaking in worms that are 5 weeks old (Wu and Loverde, 2008), which is the time at which females in mixed-sex infections begin to mature and lay eggs. HNF4 is therefore a candidate for a receptor that is able to regulate female worm mitochondrial respiration, vitellarial survival and/or fecundity.

\section{Conclusions}

Based on the available data, we have developed a model of the metabolic requirements of female schistosomes. We propose that glycolysis provides energy and intermediates for the majority of schistosome tissues, and is sufficient for survival. However, we believe that vitellocytes are highly dependent on OXPHOS and that they primarily use fatty acids acquired from their hosts to fuel this process via $\beta$-oxidation. Recent findings that female schistosomes infecting mice living on high-fat diets are fivefold more fecund than worms infecting mice being fed with regular mouse chow (Alencar et al., 2009) provide support, albeit indirect, for this view. It is feasible that, in vivo, in the absence of males, females either do not have access to, or are unable to ingest and/or absorb sufficient fatty acids to support vitellarial development. In the absence of sufficient fatty acids, the primordial vitellarial tissue could continue to create new vitellocytes by proliferation, but these cells might be unable to differentiate and survive due to a failure of $\beta$-oxidation. We hypothesize that the schistosome LD complex is functionally analogous to the insect fat body, and that in vitro the worms can continue to produce eggs until this reserve is depleted, after which egg production ceases. The regression of vitellarial tissue in cultured females even in the presence of male worms may reflect the fact that tissue culture medium is poor in key fatty acid nutrients that are available in vivo and which, in the form of short-chain and medium-chain fatty acids, are particularly well represented in portal blood versus peripheral blood (Dankert et al., 1981; Bergman, 1990). It is possible that beneficial effects of males on egg production during the initial stages of tissue culture (Michaels and Prata, 1968) may reflect their ability to help females utilize fatty acids that are present, although mechanistic details regarding how this might happen are unclear at present. Intriguingly, recent mass spectrometric analyses of host metabolic markers during infection have revealed highly significant declines in plasma short-chain fatty acid levels, which could be consistent with the extensive use of these fatty acids by the parasites (Wang et al., 2004; Balog et al., 2011). Future studies should aim to integrate vitellocyte metabolism with the role of the TGF $\beta$ signalling pathway, and other growth factor like signalling pathways, which have been shown to be important in schistosome reproduction (Freitas et al., 2007; Knobloch et al., 2007; LoVerde et al., 2009), and to explore the possible role in fatty acid oxidation of the schistosome RXR nuclear receptors, which are implicated in the regulation of expression of eggshell proteins (LoVerde et al., 2009), and homologues of which partner PPARs in vertebrates (Plutzky, 2011).

\section{Acknowledgements}

The authors' work is supported by the NIH (AI032573, Al11048 and CA164062 to EJP) and an American Heart Association Postdoctoral Fellowship to SCCH. The authors have no conflicts on interest to declare.

\section{References}

Alencar, A.C., Neves, R.H., Aguila, M.B., Mandarim-deLacerda, C.A., Gomes, D.C., and Machado-Silva, J.R. 
(2009) High fat diet has a prominent effect upon the course of chronic schistosomiasis mansoni in mice. Mem Inst Oswaldo Cruz 104: 608-613.

Armstrong, J.C. (1965) Mating behavior and development of schistosomes in the mouse. J Parasitol 51: 605-616.

Arrese, E.L., and Soulages, J.L. (2010) Insect fat body: energy, metabolism, and regulation. Annu Rev Entomo/ 55: 207-225.

Balog, C.I., Meissner, A., Goraler, S., Bladergroen, M.R., Vennervald, B.J., Mayboroda, O.A., and Deelder, A.M. (2011) Metabonomic investigation of human Schistosoma mansoni infection. Mol Biosyst 7: 1473-1480.

Barrett, J. (1981) Biochemistry of Parasitic Helminths. London: MacMillan Publishers Ltd.

Barrett, J. (2009) Forty years of helminth biochemistry. Parasitology 136: 1633-1642.

Basch, P.F. (1981) Cultivation of Schistosoma mansoni in vitro. I. Establishment of cultures from cercariae and development until pairing. J Parasitol 67: 179-185.

Basch, P.F., and Basch, N. (1984) Intergeneric reproductive stimulation and parthenogenesis in Schistosoma mansoni. Parasitology 89 (Part 2): 369-376.

Bergman, E.N. (1990) Energy contributions of volatile fatty acids from the gastrointestinal tract of various species. Physiol Rev 70: 567-590.

Berriman, M., Haas, B.J., LoVerde, P.T., Wilson, R.A., Dillon, G.P., Cerqueira, G.C., et al. (2009) The genome of the blood fluke Schistosoma mansoni. Nature 460: 352358.

Brouwers, J.F., Smeenk, I.M., van Golde, L.M., and Tielens, A.G. (1997) The incorporation, modification and turnover of fatty acids in adult Schistosoma mansoni. Mol Biochem Parasitol 88: 175-185.

Chitsulo, L., Loverde, P., and Engels, D. (2004) Schistosomiasis. Nat Rev Microbiol 2: 12-13.

Dankert, J., Zijlstra, J.B., and Wolthers, B.G. (1981) Volatile fatty acids inhuman peripheral and portal blood: quantitative determination by vacuum distillation and gas chromatography. Clin Chim Acta 110: 301-307.

Den Hollander, J.E., and Erasmus, D.A. (1984) Schistosoma mansoni: DNA synthesis in males and females from mixed and single-sex infections. Parasitology 88 (Part 3): 463476.

Don, T.A., Bethony, J.M., and Loukas, A. (2008) Saposin-like proteins are expressed in the gastrodermis of Schistosoma mansoni and are immunogenic in natural infections. Int $J$ Infect Dis 12: e39-e47.

Erasmus, D.A. (1973) A comparative study of the reproductive system of mature, immature and 'unisexual' female Schistosoma mansoni. Parasitology 67: 165-183.

Erasmus, D.A., Popiel, I., and Shaw, J.R. (1982) A comparative study of the vitelline cell in Schistosoma mansoni, $S$. haematobium, S. japonicum and S. mattheei. Parasitology 84: 283-287.

Ferreira, M.S., de Oliveira, D.N., de Oliveira, R.N., Allegretti, S.M., and Catharino, R.R. (2014a) Screening the life cycle of Schistosoma mansoni using high-resolution mass spectrometry. Anal Chim Acta 845: 62-69.

Ferreira, M.S., de Oliveira, D.N., de Oliveira, R.N., Allegretti, S.M., Vercesi, A.E., and Catharino, R.R. (2014b) Mass spectrometry imaging: a new vision in differentiating
Schistosoma mansoni strains. J Mass Spectrom 49: 86-92.

Freitas, T.C., Jung, E., and Pearce, E.J. (2007) TGF-b signaling controls embryo development in the parasitic flatworm Schistosoma mansoni. PLoS Pathog (in press).

Galanti, S.E., Huang, C.-C.S., and Pearce, E.J. (2012) Cell death and reproductive regression in female Schistosoma mansoni. PLoS Negl Trop Dis 6: e1509.

Grevelding, C.G. (2004) Schistosoma. Curr Biol 14: R545.

Guo, Y., Cordes, K.R., Farese, R.V., Jr, and Walther, T.C. (2009) Lipid droplets at a glance. J Cell Sci 122: 749752.

Gupta, B.C., and Basch, P.F. (1987) The role of Schistosoma mansoni males in feeding and development of female worms. J Parasitol 73: 481-486.

Haemmerle, G., Moustafa, T., Woelkart, G., Buttner, S., Schmidt, A., van de Weijer, T., et al. (2011) ATGL-mediated fat catabolism regulates cardiac mitochondrial function via PPAR-alpha and PGC-1. Nat Med 17: 1076-1085.

Horemans, A.M., Tielens, A.G., and van den Bergh, S.G. (1991) The transition from an aerobic to an anaerobic energy metabolism in transforming Schistosoma mansoni cercariae occurs exclusively in the head. Parasitology 102 (Part 2): 259-265.

Huang, C.-C.S., Freitas, T.C., Amiel, E., Everts, B., Pearce, E.L., Lok, S.B., and Pearce, E.J. (2012) Fatty acid oxidation is essential for egg production by the parasitic flatworm Schistosoma mansoni. PLoS Pathog 8: e1002996.

Hyman, L. (1951). The Invertebrates, Vol. 2, Platyhelminthes and Rhyncocoela. New York: McGraw Hill.

King, C.H., and Dangerfield-Cha, M. (2008) The unacknowledged impact of chronic schistosomiasis. Chronic IIIn 4: 65-79.

Knobloch, J., Kunz, W., and Grevelding, C.G. (2002) Quantification of DNA synthesis in multicellular organisms by a combined DAPI and BrdU technique. Dev Growth Differ 44: 559-563.

Knobloch, J., Beckmann, S., Burmeister, C., Quack, T., and Grevelding, C.G. (2007) Tyrosine kinase and cooperative TGFbeta signaling in the reproductive organs of Schistosoma mansoni. Exp Parasitol 117: 318-336.

Kunz, W. (2001) Schistosome male-female interaction: induction of germ-cell differentiation. Trends Parasitol 17: 227231.

LoVerde, P.T. (2002) Presidential address. Sex and schistosomes: an interesting biological interplay with control implications. J Parasitol 88: 3-13.

LoVerde, P.T., Andrade, L.F., and Oliveira, G. (2009) Signal transduction regulates schistosome reproductive biology. Curr Opin Microbiol 12: 422-428.

Meyer, F., Meyer, H., and Bueding, E. (1970) Lipid metabolism in the parasitic and free-living flatworms, Schistosoma mansoni and Dugesia dorotocephala. Biochim Biophys Acta 210: 257-266.

Michaels, R.M. (1969) Mating of Schistosoma mansoni in vitro. Exp Parasitol 25: 58-71.

Michaels, R.M., and Prata, A. (1968) Evolution and characteristics of Schistosoma mansoni eggs laid in vitro. J Parasitol 54: 921-930. 
Neves, R.H., Miranda de Barros Alencar, A.C., Costa-Silva, M., Aguila, M.B., Mandarim-de-Lacerda, C.A., Machado-Silva, J.R., and Gomes, D.C. (2007) Long-term feeding a high-fat diet causes histological and parasitological effects on murine schistosomiasis mansoni outcome. Exp Parasitol 115: 324-332.

van Oordt, B.E., van den Heuvel, J.M., Tielens, A.G., and van den Bergh, S.G. (1985) The energy production of the adult Schistosoma mansoni is for a large part aerobic. Mol Biochem Parasitol 16: 117-126.

Palanker, L., Tennessen, J.M., Lam, G., and Thummel, C.S. (2009) Drosophila HNF4 regulates lipid mobilization and beta-oxidation. Cell Metab 9: 228-239.

Pearce, E.J., and MacDonald, A.S. (2002) The immunobiology of schistosomiasis. Nat Rev Immunol 2: 499-511.

Pearce, E.L. (2010) Metabolism in T cell activation and differentiation. Curr Opin Immunol 22: 314-320.

Pellettieri, J., Fitzgerald, P., Watanabe, S., Mancuso, J., Green, D.R., and Sanchez Alvarado, A. (2010) Cell death and tissue remodeling in planarian regeneration. Dev Biol 338: $76-85$.

Plutzky, J. (2011) The PPAR-RXR transcriptional complex in the vasculature: energy in the balance. Circ Res 108: 1002-1016.

Popiel, I., and Basch, P.F. (1984a) Reproductive development of female Schistosoma mansoni (Digenea: Schistosomatidae) following bisexual pairing of worms and worm segments. J Exp Zool 232: 141-150.

Popiel, I., Cioli, D., and Erasmus, D.A. (1984b) The morphology and reproductive status of female Schistosoma mansoni following separation from male worms. Int $J$ Parasitol 14: 183-190.

Rinaldi, G., Eckert, S.E., Tsai, I.J., Suttiprapa, S., Kines, K.J., Tort, J.F., et al. (2012) Germline transgenesis and insertional mutagenesis in Schistosoma mansoni mediated by murine leukemia virus. PLoS Pathog 8: e1002820.

Rumjanek, F.D., and Simpson, A.J. (1980) The incorporation and utilization of radiolabelled lipids by adult Schistosoma mansoni in vitro. Mol Biochem Parasitol 1: 31-44.

Schiller, E.L., Bueding, E., Turner, V.M., and Fisher, J. (1975) Aerobic and anaerobic carbohydrate metabolism and egg production of Schistosoma mansoni in vitro. J Parasito/ 61: 385-389.

Shaw, M.K. (1987) Schistosoma mansoni: vitelline gland development in females from single sex infections. $J$ Helminthol 61: 253-259.

Skelly, P.J., Da'dara, A.A., Li, X.H., Castro-Borges, W., and Wilson, R.A. (2014) Schistosome feeding and regurgitation. PLoS Pathog 10: e1004246.

Wang, Y., Holmes, E., Nicholson, J.K., Cloarec, O., Chollet, J., Tanner, M., et al. (2004) Metabonomic investigations in mice infected with Schistosoma mansoni: an approach for biomarker identification. Proc Natl Acad Sci USA 101: 12676-12681.

Warburg, O. (1956) On respiratory impairment in cancer cells. Science 124: 269-270.

Wu, W., and Loverde, P.T. (2008) Schistosoma mansoni: identification of SmNR4A, a member of nuclear receptor subfamily 4. Exp Parasitol 120: 208-213.

Wu, W., and LoVerde, P.T. (2011) Nuclear hormone receptors in parasitic helminths. Mol Cell Endocrinol 334: 56-66.

Young, B.W., and Podesta, R.B. (1982) Major phospholipids and phosphatidylcholine synthesis in adult Schistosoma mansoni. Mol Biochem Parasitol 5: 165-172.

Zechner, R., Zimmermann, R., Eichmann, T.O., Kohlwein, S.D., Haemmerle, G., Lass, A., and Madeo, F. (2012) FAT SIGNALS-lipases and lipolysis in lipid metabolism and signaling. Cell Metab 15: 279-291. 